\title{
Analysis of Construction Control Technology in Construction of Long-Span Continuous Bridges
}

\author{
Huakui Yang \\ Jiangsu College of Engineering and Technology, Nantong Jiangsu, 226000
}

Keywords: Long-span Continuous Construction, Bridge Construction, Analysis and Research, Construction Control Technology

\begin{abstract}
With the development of science and technology and continuous improvement of construction technology, the continuous construction technology of long span in bridge construction has been widely used. The application of this technology has improved the reliability and safety of the bridge. The main features of construction technology include basic construction and structural construction of the upper and lower parts of the bridge. In the process of bridge construction, large-span continuous construction technology is mainly used in the construction of cable-stayed bridges, arch bridges and suspension bridges, which has achieved good results. The key to long-span continuous construction technology is linear control, stress control stability control.
\end{abstract}

\section{Introduction}

With the development of the national economy, the construction of infrastructure such as roads and bridges has become very important. In the new period of social development, the society has placed higher demands on the quality of the transportation infrastructure. The bridge construction of large-span continuous construction technology in the process improves the quality of the bridge construction and plays an important role in the development and innovation of construction technology [1]. Therefore, to strengthen the control of large span continuous construction to ensure the safety of the construction structure, so that the shape and internal force after its completion meets the bridge design requirements. This article elaborates the main contents and work flow of construction control technology for long-span continuous construction, hoping to promote the development of bridge construction [2].

\section{The Construction of Long-Span Continuous Bridge Construction Control Tasks}

\subsection{Linear control}

Linear control is mainly applied to the cantilever pouring construction, in order to ensure the overall quality of the construction, we need to do a good job of linear control work, the work carried out with the linear changes of the beam body, the deformation of the basket, the beam section, the level of prestressing, concrete creep and temperature are closely linked, linear control mainly includes linear control and longitudinal linear control two categories [1]. The main control principle is to carry out the plane linear control process to be completed in each section of the basket after the walk, through the total station to adjust the basket center line, the template reinforcement, reinforcement and then dedicated to hanging basket Line review. Longitudinal linear control requires the calculation of the vertical camber of the entire beam section, and then the pre-camber according to the measured value of the previous beam section, and the construction parameters of the next beam section should be adjusted to ensure the quality of the entire project. Hanging basket construction and design, can not only be light-weight treatment, but also take full account of its safety and operability, to achieve re-use, but also in the construction process to control the quality and strength of the basket and when completed after the hanging basket assembled, to carry out load testing to ensure that its elastic deformation within the specified range, so as to ensure the progress and quality of the bridge construction. 


\subsection{Stress Control}

In the process of continuous construction with large span, the stress control of the key cross-section of the bridge should be strengthened so that the stability and reliability of the whole structure of the bridge can be ensured in the event of potential safety hazards. The stress control technology the main working principle is to evaluate the bridge safety by analyzing and researching the calculated values and the numerous measured values [2]. The stress control should record the deviation between the calculated value and the measured value, and adjust the calculation model in time and repair, at the same time, the design parameters should be re-set to meet the bridge's stress control needs. Common means of stress monitoring detection strain method, steel wire stress calculation and reinforced stress calculation of three, it is worth mentioning that the use of steel wire stress calculation is more convenient, stable performance and less affected by the temperature, these advantages make the steel string stress calculation more suitable for long-term observations, because the temperature on the detection of work is relatively large, so the best detection in the morning to reduce the detection error.

The main work of stress control is to deal with the actual situation of the project monitoring to ensure compliance with relevant regulations. Stress control is the key technology of bridge quality management. Most workers control the stress of the whole project through the section. Therefore, before the work is carried out through the relevant test equipment to analyze the entire structure of the stress, so that we can keep abreast of the bridge stress situation in the inspection process if the stress and the provisions of the numerical deviation, it is necessary to analyze the problem, timely adjustment and solution to the problem, the error to a minimum. Bridge construction process will be a lot of poor stress, of which the work of structural stress to carry out the most difficult, even beyond the control of deformation, the main reason for the difficulty is that the problem is not easy to find, once the appearance of the entire project will have a very great impact, even serious cracks will occur, so that the entire bridge loss of force conditions.

\subsection{Stability Control}

The stability of the bridge has an important connection with the safety and quality of the bridge after its completion. It has become an important research topic in the long-span continuous construction process [3]. During the construction process, the intensity control within the structure should be strengthened. With the grasping the deformation of the structure and making timely adjustments, the stability of the bridge needs to be ensured throughout the whole project. During the construction of a special phase, it is necessary to monitor and control the stability of the bridge at each stage of construction, then manage and control the stability of the entire bridge construction. China's long-span continuous construction technology also needs to be developed and innovated. With the acceleration of the urban development process, the span of the bridge construction is getting larger and larger, which puts forward higher requirements on its stability control. The long-span continuous construction stability control, mainly through computer technology to test and analyze the internal strength of the bridge, the deformation of real-time grasp, so as to achieve the purpose of evaluating the quality of the bridge [3].

It is necessary to pay special attention to the fact that if the internal force is less than the normal value during the strength calculation, the bridge's capacity needs to be recalculated to re-estimate and review some of the major components and reduce error. The application of this technology can help the beam bridge in the process of using better maintenance, so that its load is always controlled within the prescribed range, increase the service life of the bridge, many bridges in the construction process because of its stability it is not enough for the whole bridge to be damaged. This requires strengthening the control over the stress and deformation of the bridge during the construction phase so as to ensure the stability of the components at all stages.

\subsection{Security control}

In the course of bridge construction and construction, attention should be paid to safety control. As long as the smooth construction of the project can be guaranteed, other control of the bridge can 
be made, and construction conditions can be provided for the whole bridge construction. The safety control of the construction can also be described as a summary of the above control. Stress control, stability control, linear control, and deformation control are important. Deformation control is a part of engineering construction and construction control. No matter what construction method is adopted, the bridge will be deformed. The structural deformation will be affected by many factors. It is very prone to the deviation of the position, which makes it difficult for the bridge to be closed or the linearity and design [2]. Therefore, we must ensure that the error between the actual deformation and the expected deformation during the construction process is within the acceptable range. Including the deformation control, including the above-mentioned control have been the desired control results, construction safety will be controlled, but it is worth mentioning that in the bridge construction process may occur due to quality problems caused the security accident, this situation is not counted. Due to the different construction and construction forms of the bridge, the main factors affecting the construction safety are varied. During the construction control, the actual construction situation should be investigated and mastered. The main control of the safety control should be clearly defined according to the actual situation and the construction environment Point, to carry out targeted control.

\section{The Development of Large Span Continuous Bridge Construction Control}

(1) Continuous bridge construction control is mainly to ensure the safety and reliability of the bridge structure, in line with the structural design of the stress, linear and other design requirements in the construction of the control process to develop a reasonable construction management system, control system to develop, to include kinds of technology of the observation system, and through the application of the computer to analyze the parameters obtained and processed data to be calculated, the results for the construction control of the project. With the development of construction technology and the advancement of control technology, the control of the monitoring system through computer technology has greatly improved the construction speed of bridges, and the adaptive construction methods have also begun to be widely used. Some bridge construction technologies have developed rapidly. Developed countries began to carry out construction control in the process of the sub-construction of the bridge and took construction management as a part of the construction work. The development and innovation of monitoring technology promoted the improvement of the control system, and the work flow of the control system also shifted from the traditional manual control - Pre-control analysis - Ideal state report to the new workflow development of automatic monitoring - real-time analysis - stochastic forecasting, the running process of the current large-span continuous bridge construction control system is as follows: Refer to relevant regulations and construction experience may involve the calculation of parameters, the different stages of the construction process simulation to design a linear, and then calculated in accordance with the predicted value of the stage construction, the main beam elevation, temperature, stress measurements, and then the measured value and simulation the calculated value of the comparison, the error between the two through the meter The program carries on the reasonable design, estimates the construction elevation in the next stage, then carries on the second-stage construction according to the forecast value, measures the elevation, the temperature and the stress of the main beam, repeats the above-mentioned inspection links, until all stages of the bridge construction is completed[2].

(2) In addition to construction monitoring, the use of the bridge after the completion of the construction phase has begun to get the attention of many countries, the use of the bridge monitoring refers to the bridge to establish long-term tracking monitoring equipment, this testing equipment can provide the bridge at any time. As the bridge structure continues to improve and develop, bridge construction control in China begins to develop and innovate. Compared with other countries, bridge construction control in China started relatively late, but its development speed can not be ignored. From the establishment of Lianyungang Bridge in 1982 to today, the bridge construction control technology in China has undergone tremendous changes. The domestic research on bridge construction monitoring has also made great progress. From the application of 
the original cable-stayed bridge to the current arch bridge, the research and application of suspension bridge and continuous rigid frame bridge have formed the construction control system with the characteristics of Chinese bridge construction and achieved great success [2]. With the continuous development of construction control system, various kinds of software and equipment begin to be applied in the process of construction control, but the construction environment has a great impact on its construction, so it is necessary to carry out reform and innovation. The strategy formulation of College English reading teaching should take full consideration of the students' cognitive level and choose appropriate strategies. According to the way of learning from simple to difficult, from shallow to deep strategy, we should choose easy learning strategies to mobilize students' interest in learning, and introduce difficult strategies to promote students' development.

\section{Large Span Continuous Bridge Construction Control Methods}

There are many changes in the structure of the bridge structure, the construction process of the different monitoring content there is a big difference, therefore, the bridge construction control taken by the way there are also different, the main construction control methods are adjusted afterwards control method, predictive control method, adaptive control method, maximum width tolerance method and so on [3]. Some people also take control theory as the basis of summarization. The construction control methods of bridge are divided into feedback control, open-loop control and adaptive control.

\subsection{Adjust the control method afterwards}

Post-adjustment control is generally used in the bridge structure of linear and internal force can be adjusted in the bridge, most of them are cable-stayed bridge, the bridge in the construction process once the structure deviates from the prescribed value of the situation, it is necessary to adjust the post-control way to adjust, so that the structure can meet the design requirements, this construction method in the application process there are some limitations, this method can not be applied to other bridges.

\subsection{Predictive control law}

Compared with the post-adjustment control method, the predictive control method has a wide range of applications and is suitable for construction control of all bridges. The main working principle is to predict all the possible influencing factors, and this method is used to analyze the construction objectives and after the research, the problems and the status of each construction stage may be predicted, and the construction of the bridge project will be carried out according to the prediction results [2]. This is a widely used control method in the actual construction process, especially some bridges that can not be adjusted afterwards. The predictive control method evolves according to the modern cybernetics. The methods adopted in the forecasting process are as follows: the gray system control method, stress-free state method and Kalman filter method, predictive control method also have some limitations, that is, its workload is relatively large.

\subsection{Adaptive control method}

The main objective of the application of adaptive control method is to reduce the error between the actual construction parameters and the design time parameters, because the data is not uniform, the actual structure and design requirements appear discrepancy phenomenon, in order to reduce the error, the various stages of the construction of the system to identify and calculate the parameters, and then corrected according to the calculation results, the error parameters, each stage are repeated estimates, the design requirements and the actual structure can be consistent.

\subsection{Maximum tolerance control method}

The maximum latitude is the bridge in the design process of the main beam height and stress of the error value regulation and restrictions to reduce the control of the difficulty, but it is prone to other problems, the most common is the length of the cable-stayed bridge zipper problems will 
appear , these problems will have an impact on the accuracy of the entire bridge structure, therefore, this control law still needs to be perfected.The schema theory contains a variety of reading modes, which can effectively absorb information, enhance the ability of the affair, and effectively train the students' reading consciousness[3]. Text is the communication medium between the author and the reader. Readers must fully contact the language information and background information in the text, so that we can fully understand the chill of the text and achieve the reading communicative ability. Therefore, in the process of college English teaching, we should start from the advantages of mother tongue, expand students' knowledge and enrich their knowledge of language and culture. In the process of English reading teaching, teachers should not only cultivate students' language abilities, but also increase students' vocabulary, help students understand sentences and meanings, improve reading speed and sort out text thinking.

\section{Conclusions}

In summary, it is necessary to carry out construction control work during the construction of long-span continuous bridges. Under normal circumstances, there will be some differences between the values of pre-camber in the documents and the actual construction process. Therefore, constructions carry out the design of the supplement and guidance. At the same time, the construction control should also study and test various factors that affect the construction quality and safety, and solve the problems in time. Construction control can make the deformation point of the bridge clear and fully improve the construction level of the project.

\section{References}

[1] Feng Keke. Construction technology of long-span continuous bridge in bridge construction [J]. Low Carbon World, 2016 (34): 210-211.

[2] Xiao Yuanping.Application of construction technology of long-span continuous bridge in bridge construction [J]. Journal of Science and Technology Economy, 2017 (09): 70-71.

[3] Liu Xiangyu, Xu Gao. Discussion on construction technology of long-span continuous bridge in bridge construction [J]. Journal of Highway and Transportation Scientific and Technical Information, 2017, 13 (07): 303-305. 